\title{
Structure-based Engineering of A Plant Type III Polyketide Synthase: Formation of An Unnatural Nonaketide Naphthopyrone
}

\author{
Ikuro Abe $,{ }^{*}, \dagger$ ग Hiroyuki Morita, ${ }^{\S}$ Satoshi Oguro, ${ }^{\dagger}$ Hisashi Noma,${ }^{\dagger}$ Kiyofumi Wanibuchi,${ }^{\dagger}$ \\ Nobuo Kawahara, ${ }^{\ddagger}$ Yukihiro Goda, ${ }^{\ddagger}$ Hiroshi Noguchi, ${ }^{\dagger}$ Toshiyuki Kohno*,$\$$ \\ $\dagger$ School of Pharmaceutical Sciences, University of Shizuoka, Shizuoka 422-8526, Japan \\ I PRESTO, Japan Science and Technology Agency, Kawaguchi, Saitama 332-0012, Japan \\ $\$$ National Institute of Health Sciences (NIHS), Kamiyoga, Tokyo 158-8501, Japan \\ $\S$ Mitsubishi Kagaku Institute of Life Sciences (MITILS), Machida, Tokyo 194-8511, Japan
}

2-Acetonyl-8,10-dihydroxy-5-methyl-4H-naphtho[1,2-b]pyran-4-one: ${ }^{1} \mathrm{H}$ NMR (800 MHz, $\left.\mathrm{CD}_{3} \mathrm{OH}\right): \delta 7.20(\mathrm{~s}, 1 \mathrm{H}), 6.60(\mathrm{~s}, 1 \mathrm{H}), 6.52(\mathrm{~s}, 1 \mathrm{H}), 6.31(\mathrm{~s}, 1 \mathrm{H}), 3.94(\mathrm{~s}, 2 \mathrm{H}), 2.77$ (s, 3H), $2.34(\mathrm{~s}, 3 \mathrm{H})$. ${ }^{13} \mathrm{C}$ NMR (200 MHz, $\mathrm{CD}_{3} \mathrm{OH}$ ): $\delta$ 204.6, 181.6, 161.8, 161.4 158.9, 140.7, 135.8, 126.7, 117.5, 114.7, 108.3, 104.2, 102.8, 48.1, 30.2, 23.3. UV: $\lambda_{\max } 240,272,360 \mathrm{~nm}$. LRMS (FAB): $m / z, 299[\mathrm{M}+\mathrm{H}]^{+}, 277$, 185, 93, 75. HRMS (FAB): found for $\left[\mathrm{C}_{17} \mathrm{H}_{15} \mathrm{O}_{5}\right]^{+}$299.0893; calcd. 299.0920. Comparative NMR $\left({ }^{1} \mathrm{H}\right.$ and ${ }^{13} \mathrm{C}$ NMR) data for the novel nonaketide and aloesone (2-acetonyl-7-hydroxy-5-methylchromone) (Che, Q. M.; Akao, T.; Hattori, M.; Kobayashi, K.; Namba, T. Chem. Pharm. Bull. 1991, 39, 704-708).

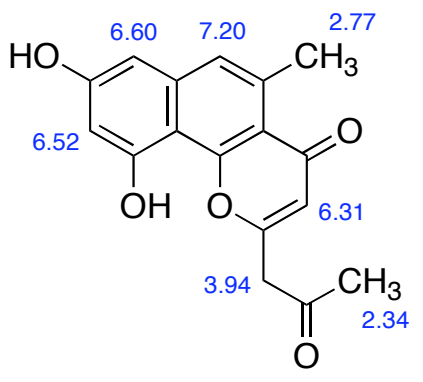

2-acetonyl-8, 10-dihydroxy-5-methyl-4H-naphtho[1,2-b]pyran-4-one $\left(\mathrm{CD}_{3} \mathrm{OH}\right)$

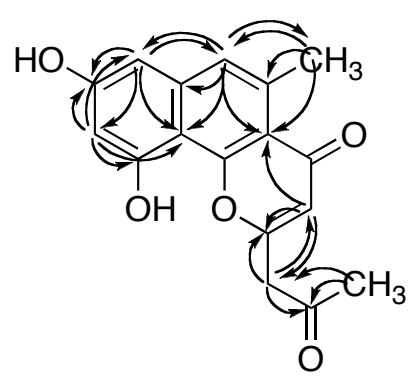

HMBC correlation<smiles>CC(=O)Cc1cc(=O)c2c(C)cc(O)cc2o1</smiles>

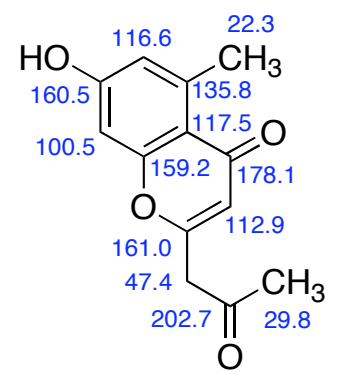

aloesone (2-acetonyl-7-hydroxy-5-methylchromone) (DMSO- $\mathrm{d}_{6}$ ) 


\section{${ }^{1} \mathrm{H}-\mathrm{NMR}$}

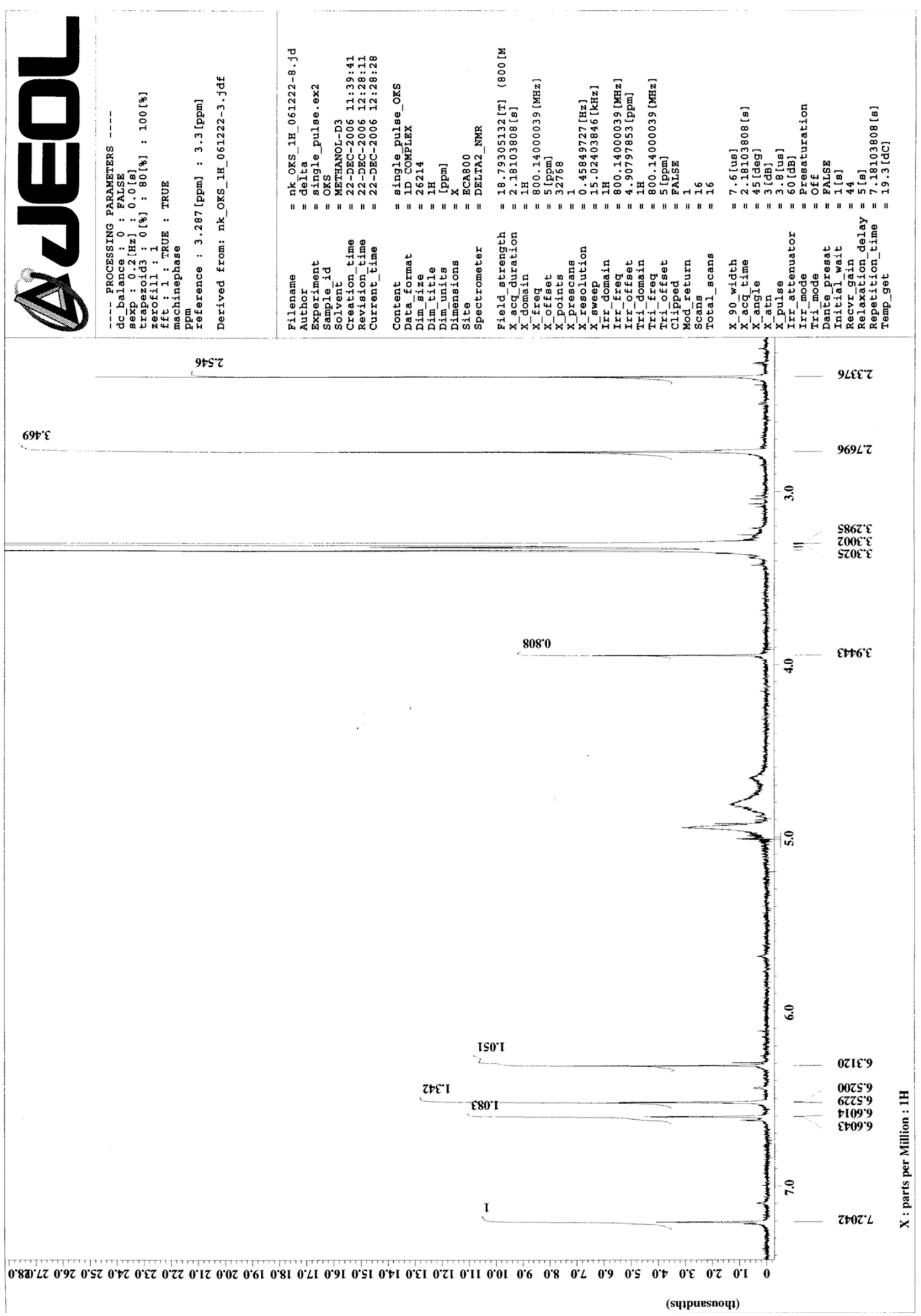




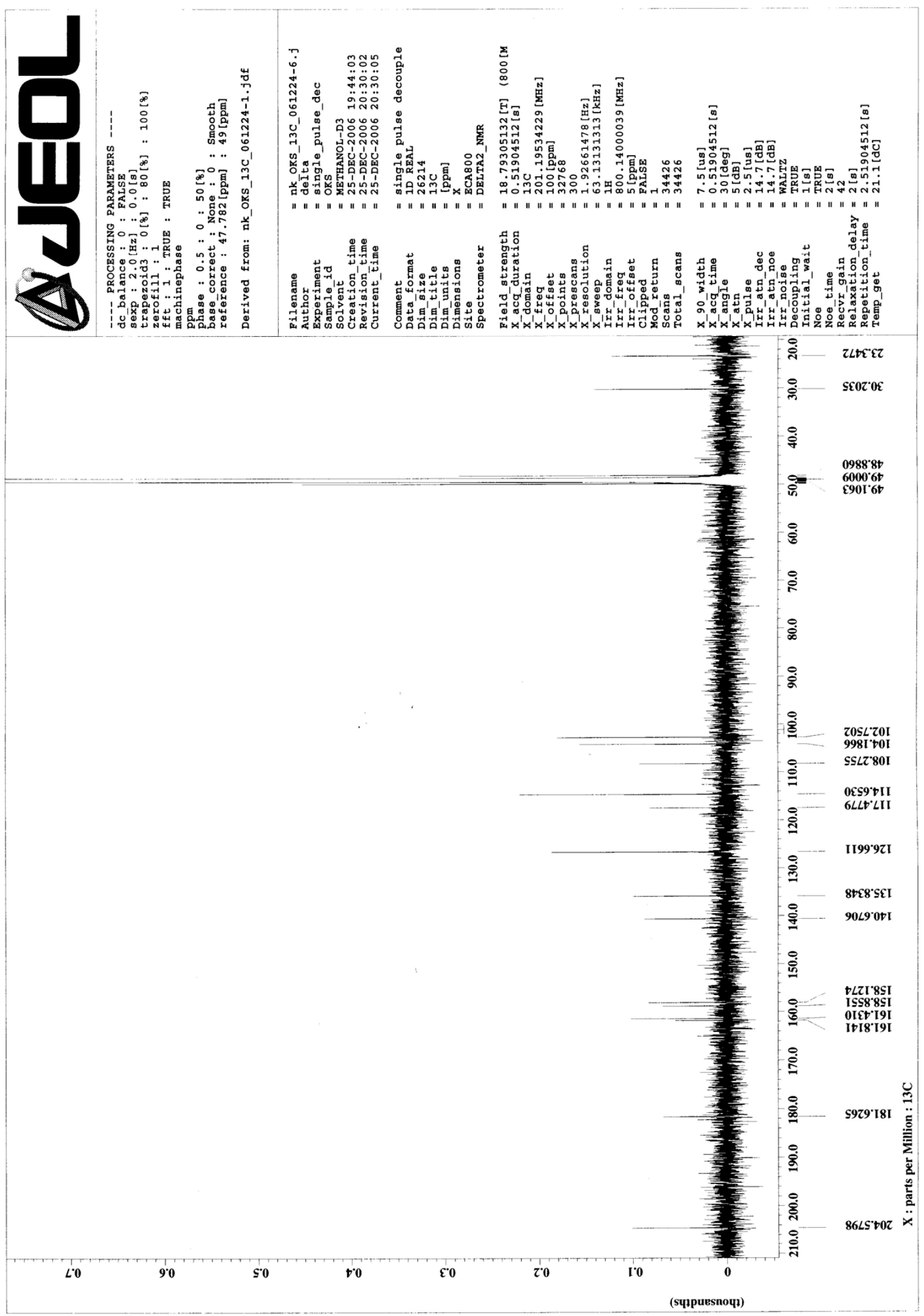




\section{DEPT135}

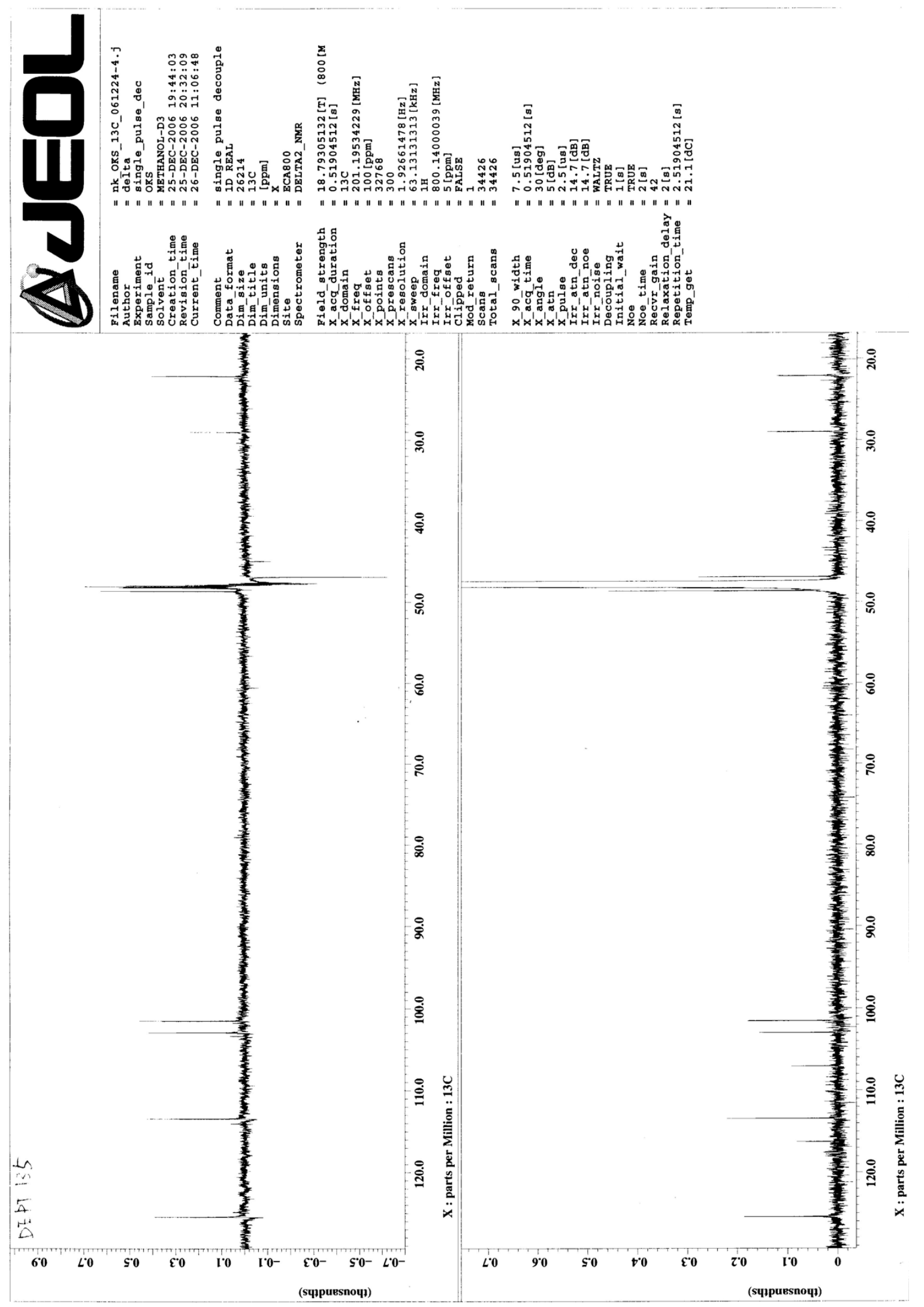




\section{HMQC}

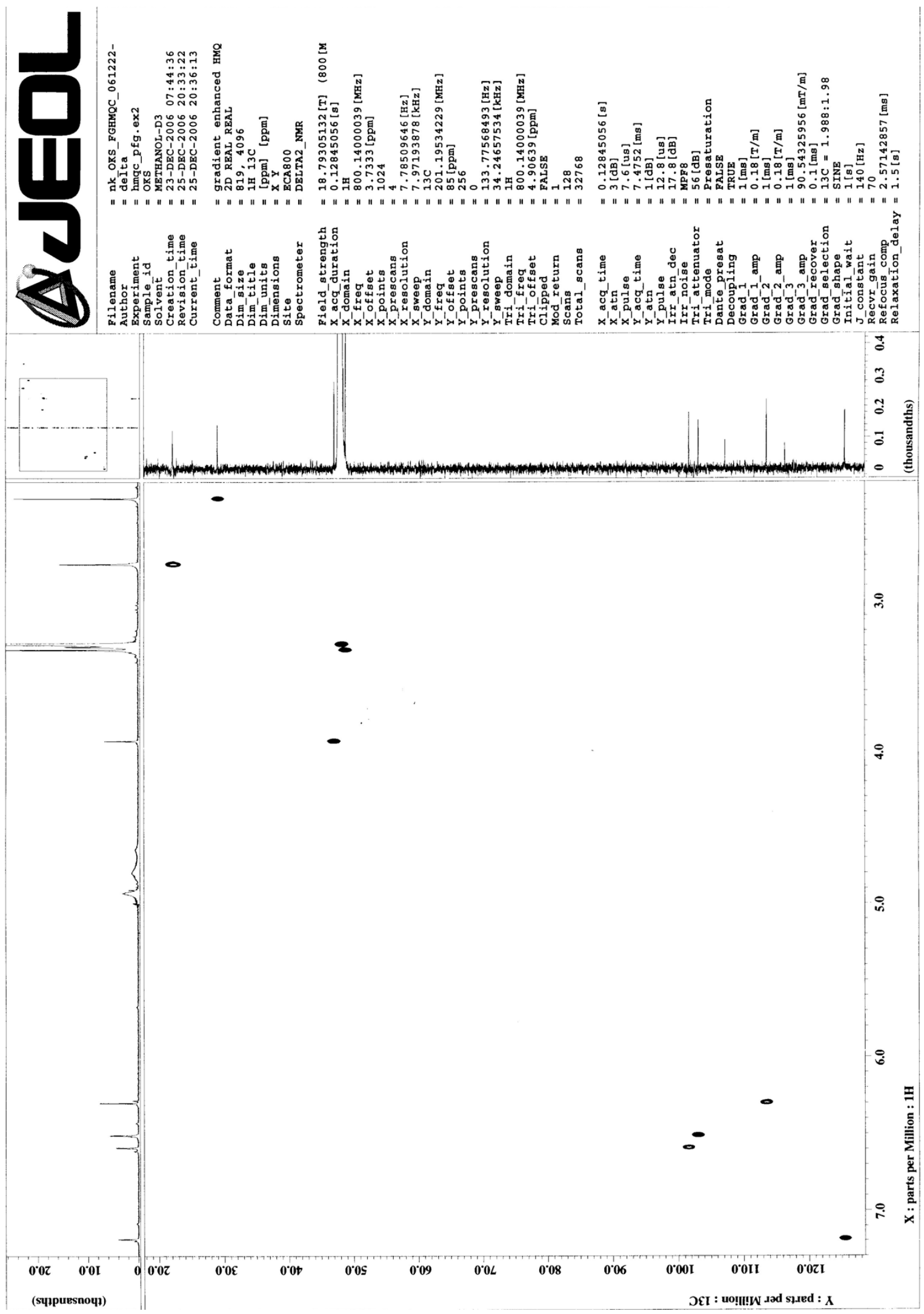


HMBC (1)

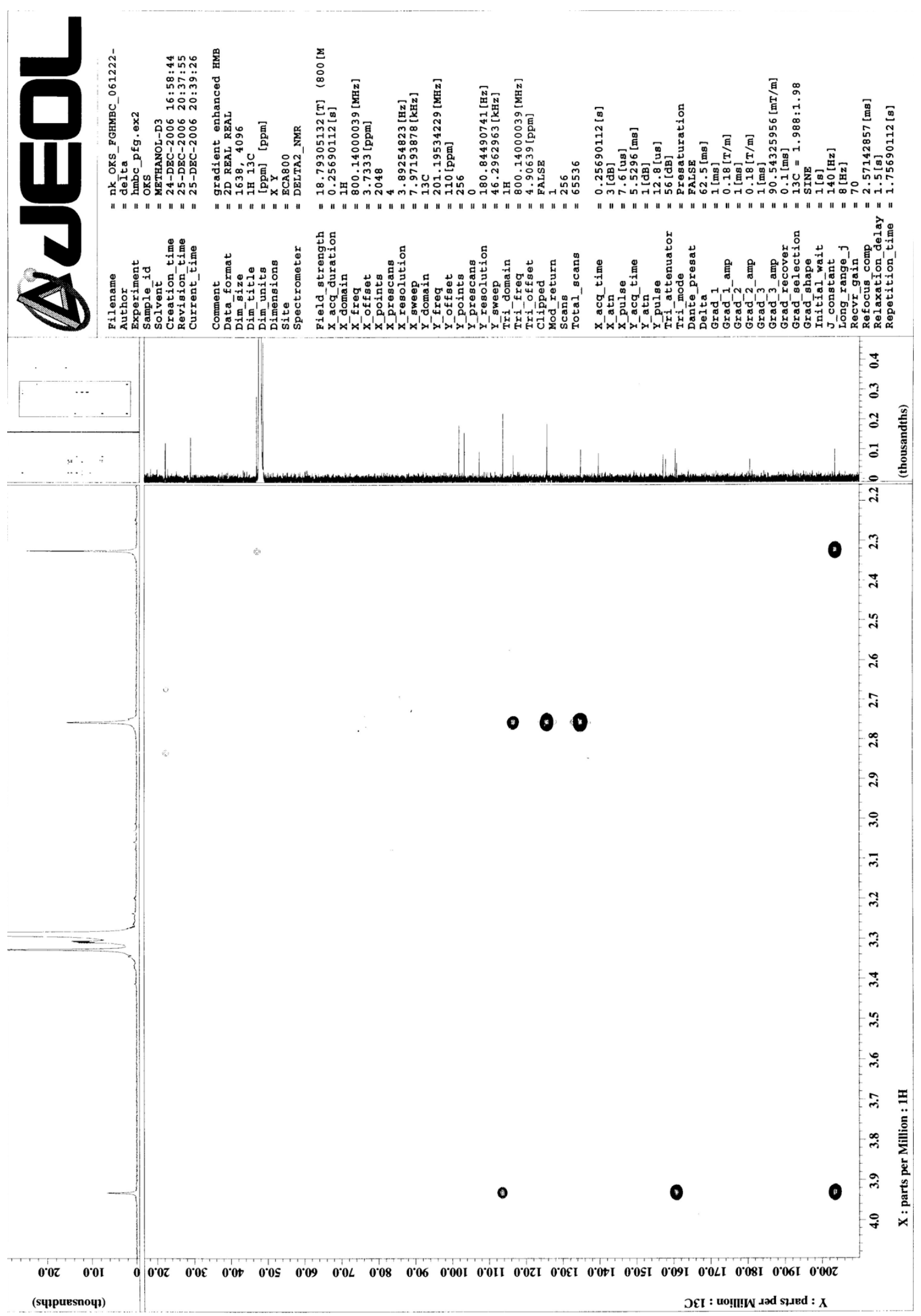


HMBC (2)

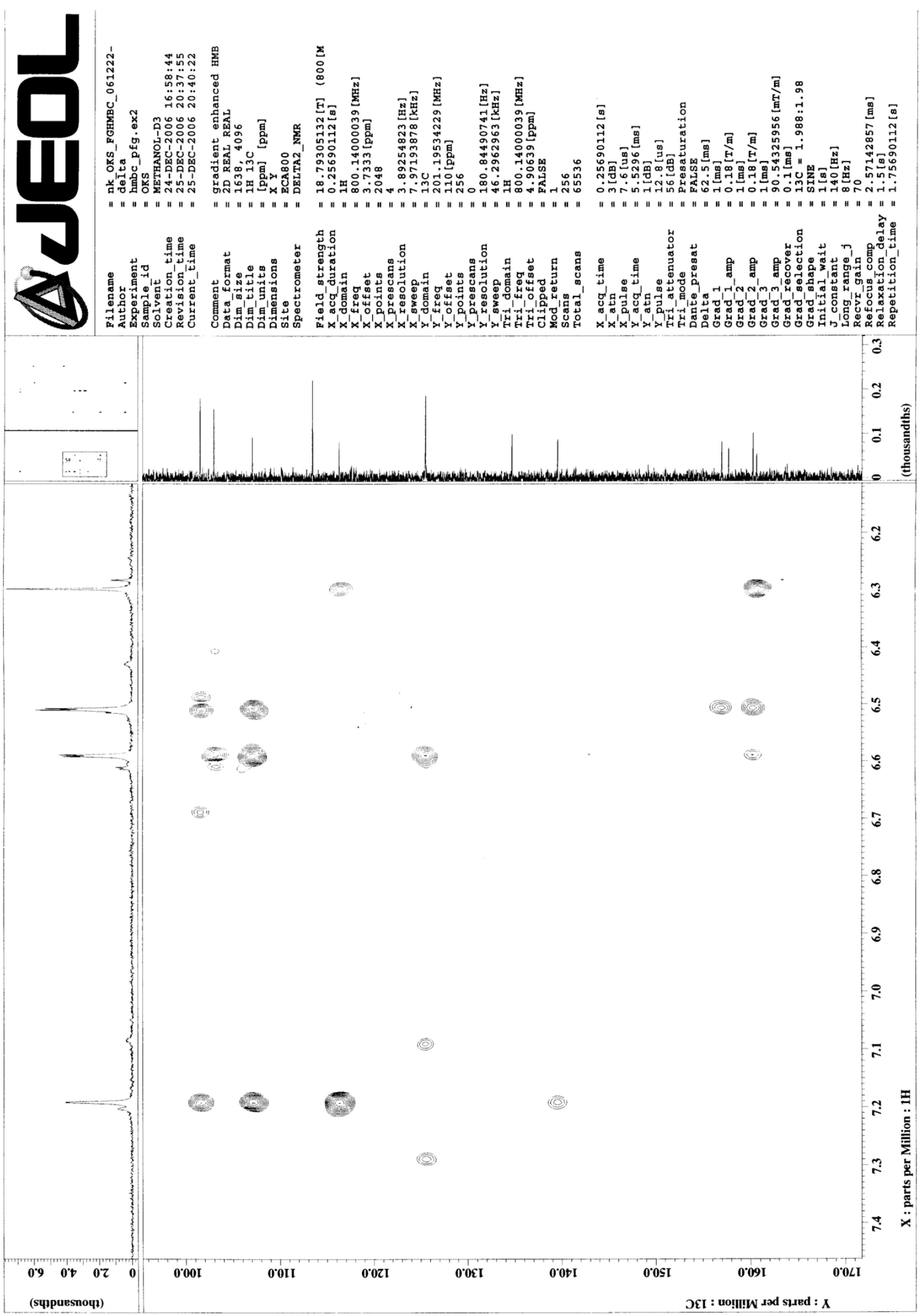


HMBC (3)

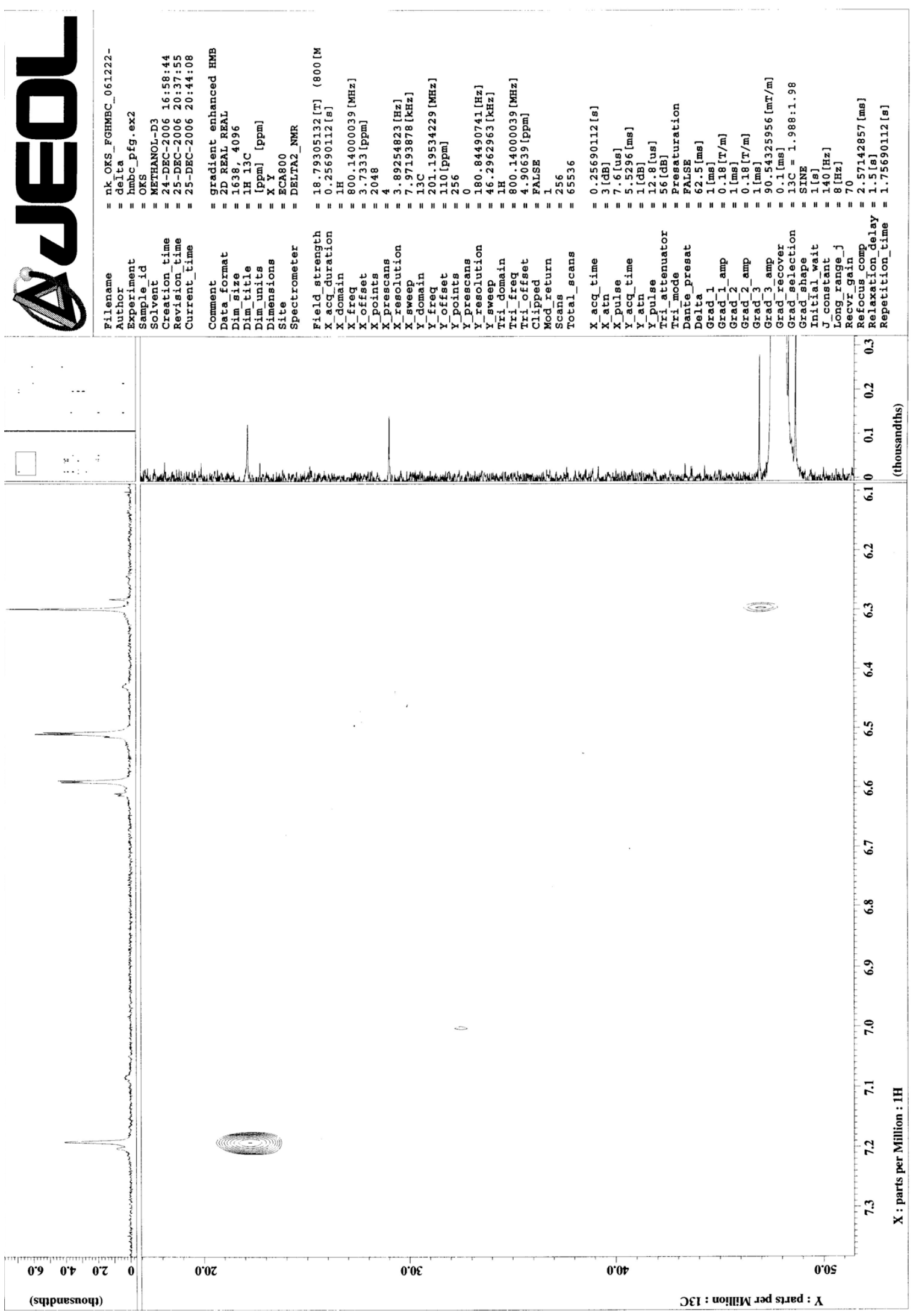

